\title{
Rough-conduit flows and the existence of fully developed turbulence
}

\author{
G. Gioia and Pinaki Chakraborty \\ Department of Theoretical and Applied Mechanics, University of Illinois, Urbana, IL 61801 \\ Fabián A. Bombardelli \\ Department of Civil and Environmental Engineering, University of California, Davis, CA 95616
}

\begin{abstract}
It is widely believed that at high Reynolds number $(\mathrm{Re})$ all turbulent flows approach a state of "fully developed turbulence" defined by a unique, Re-independent statistics of the velocity fluctuations. Yet direct measurements of the velocity fluctuations have failed to yield clear-cut empirical evidence of the existence of fully developed turbulence. Here we relate the friction coefficient $(f)$ of rough-conduit flows to the statistics of the velocity fluctuations. In light of experimental measurements of $f$, our results yield unequivocal evidence of the existence of fully developed turbulence.
\end{abstract}

Turbulence is the unrest that spontaneously takes over a streamline flow when the flow is made sufficiently fast. A conspicuous manifestation of this unrest, and one that lends itself readily to theoretical analysis, is the advent of fluctuations in the velocity field of the flow. In a classic paper 1], Kolmogórov made a few plausible assumptions to show that in flows of high mean velocity (or high Re) the statistics of the velocity fluctuations should become asymptotically invariant to further increases in the mean velocity. The universal turbulent state defined by the limiting statistics of the velocity fluctuations was termed "fully developed turbulence," and its existence has become a widely held belief. Support for this belief has been sought in direct, hot-wire measurements of the velocity fluctuations; yet, as recent research has shown 2, 3, 4, 5], the results remain inconclusive. In this Letter, we seek to prove the existence of fully developed turbulence by harnessing empirical evidence other than the direct measurement of the velocity fluctuations. We start with an outline of the intricacies of the problem.

Kolmogórov studied the statistics of the velocity fluctuation at the lengthscale $l, u_{l}$. He used dimensional analysis to show that the structure function $\overline{\left(u_{l}\right)^{2}}$ (i.e., the mean value of $\left.\left(u_{l}\right)^{2}\right)$ must take the form [1]

$$
\overline{\left(u_{l}\right)^{2}}=P[\operatorname{Re}, l / L](\varepsilon l)^{2 / 3},
$$

where $P$ is a dimensionless function of the dimensionless variables $\operatorname{Re}$ and $l / L, \operatorname{Re} \gg 1$ is a Reynolds number of the flow, $L$ is the largest lengthscale in the flow, $\varepsilon$ is the mean value of the rate of energy dissipation per unit mass, and $l$ is confined to the inertial range, $L \gg l \gg \eta$, where $\eta$ is the viscous (dissipation) lengthscale $[6]$. Given that $\operatorname{Re} \gg 1$ and $l / L \ll 1$, it is natural to identify a plausible asymptotic scenario for $\operatorname{Re} \rightarrow \infty$ and $l / L \rightarrow 0$. To that end, Kolmogórov assumed complete similarity with respect to Re and $l / L$, or $\lim _{\operatorname{Re} \rightarrow \infty} \lim _{l / L \rightarrow 0} P=p$, where $p>0$ is a constant prefactor 7]. Under this assumption, the leading term in the asymptotic expansion of $\overline{\left(u_{l}\right)^{2}}$ is $\overline{\left(u_{l}\right)^{2}}=p(\varepsilon l)^{2 / 3}$ (where the subscript "lt" stands for leading term), which is independent of Re, and may therefore be said to define a universal limiting state for Re $\gg 1$ - the state of fully developed turbulence 8]. Shortly after the publication of Kolmogórov's paper in 1941, it was objected that the asymptotic scenario proposed by Kolmogórov, and customarily known as K41, could not account for intermittency-a phenomenon whereby the rate of energy dissipation per unit mass fluctuates around its mean value, $\varepsilon$ [9]. To account for intermittency, Kolmogórov himself argued that the similarity with respect to $l / L$ might be incomplete, and went on to assume the simplest type of incomplete similarity with respect to $l / L$ [] . Under this assumption, the leading term of $\overline{\left(u_{l}\right)^{2}}$ is $\overline{\left(u_{l}\right)^{2}}{ }_{\mathrm{lt}}=p(\varepsilon l)^{2 / 3}(l / L)^{\alpha}$, where $\alpha$ is a constant intermittency exponent [10]. This alternative asymptotic scenario, known as K62, has led to a vast body of research on intermittency 9]. Nevertheless, in K62 $\overline{\left(u_{l}\right)^{2}}$ lt remains independent of Re, as was the case in $\mathrm{K} 41$, and therefore continues to define a universal limiting state for Re $\gg 1$. In this sense, K62 represents only a minor departure from K41. More recently, a major departure from K41 has been suggested on the basis of new experimental results [2, 3]. These results indicate that even at very high Re the prefactor $p$ is not constant, but subject to a discernible dependence on $\mathrm{Re}$ - a dependence that is marked enough as to cast doubts on the existence of fully developed turbulence. To analyze these results, Barenblatt and Goldenfeld [4] argued that there might be no similarity with respect to Re. Further, they argued that the form of the structure function at high Re should be invariant under a natural redefinition of Re [1], and showed that for this principle of asymptotic covariance to hold the structure function (and therefore $p$ ) must depend on Re only through $\ln$ Re. Last, they wrote $p(\ln \operatorname{Re})=p_{0}+p_{1} \delta+\mathrm{o}(\delta)$, where $\delta \equiv 1 /|\ln \operatorname{Re}| \ll 1[12]$. Under these conditions,

$$
\overline{\left(u_{l}\right)^{2}}=\left(p_{0}+\frac{p_{1}}{\ln \operatorname{Re}}\right)(\varepsilon l)^{2 / 3}(l / L)^{\alpha}+\mathrm{o}\left(\frac{1}{\ln \operatorname{Re}}\right),
$$

and the existence of a universal limiting state depends on the value of $p_{0} \geq 0$. If $p_{0}>0$, then $\overline{\left(u_{l}\right)^{2}}=$ $p_{0}(\varepsilon l)^{2 / 3}(l / L)^{\alpha}$, which is independent of Re, and a universal limiting state exists, identical with that predicted 
by K41 (if $\alpha=0$ ) or K62 (if $\alpha \neq 0$ ) [13]. On the other hand, if $p_{0}=0$, then $\overline{\left(u_{l}\right)^{2}}{ }_{\mathrm{lt}}=p_{1}(\varepsilon l)^{2 / 3}(l / L)^{\alpha} / \ln \mathrm{Re}$, which depends on $R e$, and a universal limiting state does not exist. To decide between these alternative scenarios, Barenblatt and Goldenfeld computed best fits of (2) to hot-wire data from a large wind tunnel and the atmosphere 2], both for $p_{0}>0$ and $p_{0}=0$, and concluded that the data were "not inconsistent with either of the two possibilities" [4]. In another attempt at deciding the matter, Sreenivasan [5] studied a large set of hot-wire data, and concluded that the prefactor $p$ is "more or less universal, essentially independent of the flow as well as the Reynolds number" 14]. Nevertheless, he noted that the scatter in the data was large, and that to evince the behavior of $p$ at high Re one would have "to cover a wide range of Reynolds numbers in a single, well-controlled flow, and use instrumentation whose resolving power and quality remains equally good in the entire range;" unfortunately, "such experiments and efforts are not yet in the horizon at present" [5]. To decide the matter, we intend to resort to experimental data on the friction coefficient of rough conduits, $f$. These data (e.g., 15]) appear to be well suited to our purpose: they contain very little scatter and show beyond doubt that, for any fixed wall roughness, the leading term of $f$ is independent of Re at high Re. Further, the observed dependence of the leading term of $f$ on the roughness is described accurately by a well-known empirical formula. The only problem, to which we turn presently, is how to relate $f$ to $\overline{\left(u_{l}\right)^{2}}$.

The friction coefficient of a conduit of circular crosssection may be defined as $f \equiv \tau / \rho V^{2}$, where $\tau$ is the shear stress on the wall of the conduit, $\rho$ the density of the liquid flowing through the conduit, and $V$ the average velocity of the flow. We seek to obtain an expression relating $f$ to the structure function of (2). Now (2) was originally derived under the assumptions of isotropy and homogeneity, but the turbulent flow in a conduit is both anisotropic and inhomogeneous. Nevertheless, recent research [16] has established that (2) applies as well to flows that are neither isotropic nor homogeneous. Further, if $v_{l}$ denotes the characteristic velocity of a turbulent eddy of size $l$, we may identify $v_{l}=\sqrt{\overline{\left(u_{l}\right)^{2}}}$, where $\overline{\left(u_{l}\right)^{2}}$ is given by (2) with $L=D$ (the diameter of the conduit, which sets the largest lengthscale in the flow) (9], chap. 7). Thus, it follows from (2) that, regardless of the value of $p_{0}$, the smaller the eddy the lower its velocity. With these considerations in mind, we now seek to derive an expression for $\tau$, the shear stress on the rough wall of the conduit.

Let us call $S$ the wetted surface tangent to the peaks of the roughness elements of the wall, Fig. 1 (We assume roughness elements of uniform size $r$, as in Nikuradse's experiments 15].) Then, for $\operatorname{Re} \gg 1$, the shear stress is effected by momentum transfer across $S$. Above $S$, the velocity of the flow scales with $V$, and the fluid carries

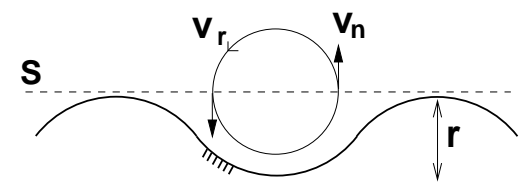

FIG. 1: Immediate vicinity of a the rough-conduit wall with roughness elements of uniform size $r$ [25]. The dashed line is the trace of the wetted surface $S$.

a high horizontal momentum per unit volume $(\sim \rho V)$. Below $S$, the velocity of the flow is negligible, and the fluid carries a negligible horizontal momentum per unit volume. Now consider an eddy that straddles the wetted surface $S$ (one half of the eddy is above $S$, the other half below). This eddy transfers fluid of high horizontal momentum downwards across $S$, and fluid of negligible horizontal momentum upwards across $S$. The net rate of momentum transfer across $S$ is set by the velocity normal to $S$, which velocity is provided by the eddies that straddle $S$. Therefore, if $v_{n}$ denotes the velocity normal to $S$ provided by the dominant eddy that straddles $S$, then the shear stress effected by momentum transfer across $S$ scales in the form $\tau \sim \rho V v_{n}$. Now the size of the largest eddy that straddles $S$ scales with $r$, the size of the roughness elements. This eddy provides a velocity $v_{r}=\sqrt{\overline{\left(u_{r}\right)^{2}}}$ normal to $S$, where $\overline{\left(u_{r}\right)^{2}}$ is given by (2) with $l=r$ and $L=D$. Smaller eddies do provide a velocity normal to $S$, but these velocities are overwhelmed by the velocity of the eddy of size $r$. Thus, $v_{n} \sim v_{r}$, and the dominant eddy that straddles $S$ is the largest eddy that straddles $S$. We conclude that $\tau \sim \rho v_{r} V$, and therefore $f \sim v_{r} / V=\sqrt{\overline{\left(u_{r}\right)^{2}}} / V$.

To complete our derivation, we relate $\varepsilon$ to $V$ and $D$ using the phenomenological theory, which is based on two tenets pertaining to the steady production of turbulent (kinetic) energy: (1) The production occurs at the lengthscale of the largest eddies in the flow and (2) The rate of production is independent of the viscosity. From these tenets and the equality of production and dissipation, it follows that we can obtain a scaling expression for $\varepsilon$, the rate of dissipation of turbulent energy per unit mass of liquid, in terms of the velocity of the largest eddies (which $\sim V$ ) and of the size of the largest eddies (which $\sim D$ ) [17]. The largest eddies possess a kinetic energy per unit mass $e \sim V^{2}$ and a turnover time $t \sim D / V$. These eddies persist for a time $t$, whereupon they split into eddies of size $\sim D / 2$, thereby transferring their energy to smaller lengthscales. For the steady state to be preserved, a new set of large eddies must be produced at time intervals $t$, implying that $\varepsilon=e / t \sim V^{3} / D$ [18]. By using $\varepsilon \sim V^{3} / D, l=r$, and $L=D$ in (2), and substituting the result in $f \sim \sqrt{\overline{\left(u_{r}\right)^{2}}} / V$, we obtain

$$
f \sim(r / D)^{1 / 3+\alpha / 2}\left(p_{0}+\frac{p_{1}}{\ln \operatorname{Re}}\right)^{1 / 2},
$$




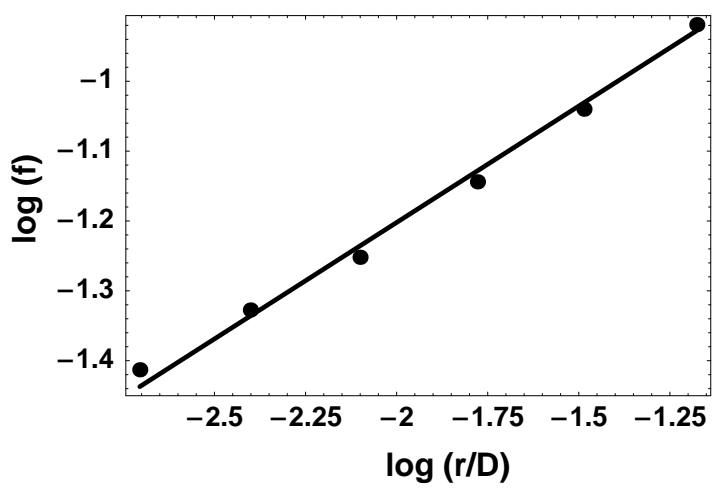

FIG. 2: A comparison of Nikuradse's data with Strickler's expression. The straight line is $\log (f)=-0.54+\log (r / D) / 3$. The data points correspond to the highest $\operatorname{Re}\left(\approx 10^{6}\right)$ tested by Nikuradse.

an expression relating $f$ to the parameters $p_{0}, p_{1}$, and $\alpha$ of the asymptotic expansion of the structure function.

Now consider a conduit of fixed roughness, $r / D=$ const $\ll 1$. If $p_{0}=0$ the leading term of $f$ depends on Re: $f_{1 \mathrm{t}} \sim \sqrt{p_{1}}(r / D)^{1 / 3+\alpha / 2}(\ln \operatorname{Re})^{-1 / 2}$. Thus, for $p_{0}=0$ the friction coefficient vanishes asymptotically at high Re, a conclusion that is at odds with all experimental data on rough-conduit flows. On the other hand, if $p_{0}>0$ the leading term of $f$ is independent of Re: $f_{\mathrm{lt}} \sim \sqrt{p_{0}}(r / D)^{1 / 3+\alpha / 2}$. Thus, for $p_{0}>0$ the friction coefficient tends to a positive constant at high Re, a conclusion that is qualitatively consistent with all experimental data on rough-conduit flows. Further, in the case $\alpha=0$, $f_{\mathrm{lt}} \sim \sqrt{p_{0}}(r / D)^{1 / 3}$, which we recognize as Strickler's empirical expression for the friction coefficient of a conduit of roughness $r / D$ at high $\operatorname{Re}[19,20]$. In the case $\alpha \neq 0$, $f_{\mathrm{lt}} \sim \sqrt{p_{0}}(r / D)^{1 / 3+\alpha / 2}$, which is a generalized form of Strickler's empirical expression that accounts for the effect of intermittency. Given that the experimental data can be fitted very well even if $\alpha$ is set to zero [Fig. 2], we infer that $1 / 3 \gg|\alpha| / 2$, or $|\alpha| \ll 2 / 3$, consistent with the available estimates of $\alpha$ 12.

From the previous paragraph, we conclude that equation (3) together with the experimental data embodied by Strickler's empirical expression allows us to establish that $p_{0}>0$. Therefore, the leading term of the structure function $\overline{\left(u_{l}\right)^{2}}$ is independent of Re, and the universal limiting state known as fully developed turbulence exists at high Reynolds numbers.

The logic of our reasoning so far has been the following. We have shown theoretically that in a rough conduit $\lim _{R e \rightarrow \infty} f=0$ unless $p_{0}>0$. In other words, we have shown that $p_{0}>0$ is a necessary condition for the friction coefficient of a rough conduit to tend to a positive constant at high Re. Then, given the unequivocal experimental evidence that in a rough conduit $\lim _{\operatorname{Re} \rightarrow \infty} f=$ const $>0$, we have concluded that it must be that $p_{0}>0$. Interestingly, $p_{0}>0$ is not in general a sufficient condition for the friction coefficient of a conduit to tend to a positive constant at high Re: the conduit must be rough. To elucidate this statement, we recall our scaling expression for the shear stress, $\tau \sim \rho v_{r} V$. This expression indicates that the momentum transfer is dominated by the eddies of size $r$ - the same size as the roughness elements. Now in a rough-conduit flow of sufficiently high Re, $r$ exceeds the viscous lengthscale, $r>\eta$. In fact, from $\eta \equiv \nu^{3 / 4} \varepsilon^{-1 / 4}$ (where $\nu$ is the kinematic viscosity) and $\varepsilon \sim V^{3} / D$ we can write $\eta / D \sim(\nu / V D)^{3 / 4}=\operatorname{Re}^{-3 / 4}$ and conclude that for any given $r$ the condition $r>\eta$ holds at sufficiently high Re. If Re is increased further, $\eta$ lessens and newer, smaller eddies populate the flow and become jumbled with the older eddies. Yet the momentum transfer continues to be dominated by eddies of size $r$, and $f$ remains invariant. This argument explains the behavior of $f$ for all rough conduits, no matter how small the the roughness elements. If $r=0$, however, the condition $r>\eta$ cannot be attained, even at extremely high Re. Thus in a smooth conduit 21] the momentum transfer will always be dominated by the smaller eddies in the inertial range, whose size scales with $\eta$; since $\eta$ lessens as Re increases, the momentum transfer will be dominated by ever smaller eddies as Re increases, and $\lim _{R e \rightarrow \infty} f=0$ - even though $p_{0}>0$, and the turbulence will be fully developed at high Re. To verify this conclusion mathematically, we study (3) for $r \sim \eta$. By substituting $\eta / D \sim \mathrm{Re}^{-3 / 4}$ in place of $r / D$, we obtain

$$
f \sim \operatorname{Re}^{-1 / 4-3 \alpha / 8}\left(p_{0}+\frac{p_{1}}{\ln \operatorname{Re}}\right)^{1 / 2} .
$$

In accord with our discussion above, (4) indicates that in a smooth conduit the friction coefficient vanishes asymptotically at high Re, whether $p_{0}=0$ or $p_{0}>0$. Had we to decide between $p_{0}=0$ and $p_{0}>0$ on the basis of (4) and experimental data, the answer would not be clearcut, because $(\ln R e)^{-1 / 2}$ varies but very slowly at high Re. Nevertheless, we have established previously that $p_{0}>0$. It follows that $f_{\mathrm{lt}} \sim \sqrt{p_{0}} \mathrm{Re}^{-1 / 4-3 \alpha / 8}$, which in the case $\alpha=0$ coincides with Blasius's empirical expression for the friction coefficient of a smooth conduit at high $\operatorname{Re}[20]$. In the case $\alpha \neq 0, f_{\mathrm{lt}} \sim \sqrt{p_{0}} \operatorname{Re}^{-1 / 4-3 \alpha / 8}$ is a generalized form of Blasius's empirical expression that accounts for the effect of intermittency.

From the previous paragraph, we conclude that if $p_{0}>$ 0 (as we established before), our theoretical predictions are in accord with the experimental data embodied by Blasius's empirical expression.

In a recent monograph 22], Tsinober has drawn a distinction between "possibly universal properties of small scale turbulence" and "quantitative universal properties of turbulence at high Re" 14]. The former were first envisioned in the early history of turbulence physics; they are embodied by the asymptotic scenarios of Kolmogórov, $\mathrm{K} 41$ and $\mathrm{K} 62$, that define the possibly universal limiting state of fully developed turbulence. The latter have 
long been known to engineers; they are manifest in a few properties of turbulent flows that become independent of Re at high Re, including the drag of bluff bodies and the friction coefficient of rough conduits, among others 23. Here we have established a relation between the two [24]. In particular, we have found that the existence of fully developed turbulence is a necessary condition for the friction coefficient of rough conduits to tend to positive constants at high Re, as seen in experiments. In addition, we have found that the existence of fully developed turbulence is compatible with the experimental evidence that the friction coefficient of smooth conduits tends to zero at high Re. On the basis of our findings, fully developed turbulence may be termed a "universal property of small scale turbulence," without qualification.

We thank J. W. Phillips for kindly reading our manuscript and suggesting ways of improving it.

[1] Reprinted in English in A. N. Kolmogórov, Proc. R. Soc. London A434, 9 (1991).

[2] A. Praskovsky and S. Oncley, Phys. Fluids 6, 2886 (1994).

[3] L. Mydlarski and Z. Warhaft, J. Fluid Mech. 320, 331 (1996) and L. Mydlarski and Z. Warhaft, ibidem 358, 135 (1998).

[4] G. I. Barenblatt and N. Goldenfeld Phys. Fluids 7, 3078 (1995).

[5] K. R. Sreenivasan, Phys. Fluids 7, 2778 (1995).

[6] A similar expression holds for the structure function of order $n, \overline{\left(u_{l}\right)^{n}}=P_{n}[\operatorname{Re}, l / L](\varepsilon l)^{n / 3}$, but we need concern ourselves only with the structure function of order 2 .

[7] G. I. Barenblatt, Scaling, Self-similarity, and Intermediate Asymptotics, ch. 10 (Cambridge University Press, Cambridge, UK, 1986).

[8] Barenblatt and Goldenfeld wrote "The mathematical expression of the assumption of a limiting state of fully developed turbulence is that statistical averages of the flow exhibit complete similarity with respect to Re" [4].

[9] U. Frisch, Turbulence (Cambridge University Press, Cambridge, UK, 1995).

[10] A. N. Kolmogórov, J. Fluid Mech. 13, 82 (1962).

[11] For example, the form of the structure function should not change if we used the Taylor microscale Reynolds number, $\mathrm{Re}_{\lambda}$, in lieu of a global Re.

[12] Barenblatt and Goldenfeld also assumed a general type of incomplete similarity with respect to $l / L$ in which the intermittency exponent, $\alpha$, may depend on Re [7]. Then, in keeping with the principle of asymptotic covariance, they wrote $\alpha(\ln \operatorname{Re})=\alpha_{0}+\alpha_{1} / \ln \operatorname{Re}+\mathrm{o}(1 / \ln \operatorname{Re})$, and pointed out that the experimental data of Praskovsky and Oncley [2] favor the case $\alpha_{0}=0$, which implies that $\alpha$ tends to zero at high Re. Since $\alpha$ is of slight concern here, we continue to represent it as a constant. Nevertheless, in all our equations $\alpha$ may be substituted by $\alpha_{0}+\alpha_{1} / \ln \operatorname{Re}+\mathrm{o}(1 / \ln \operatorname{Re})$. Note that our conclusions do not depend on the satisfaction of the principle of asymptotic covariance; in fact, they would still hold if, say, $p(\operatorname{Re})=p_{0}+p_{1} \operatorname{Re}^{-\beta}$, as has been proposed by
Y. Tsuji, Phys. Fluids 16, L43 (2004). For experiments that support the principle of asymptotic covariance see A. Arneodo, S. Manneville, J. F. Muzy and S. G. Roux, Phil. Trans. R. Soc. Lond. A 357, 2415 (1999) and G. I. Barenblatt and A. J. Chorin, PNAS 101, 15023 (2004).

[13] Note that if $p_{0}>0$ and $p_{1} \neq 0$ we continue to say that fully developed turbulence exists, even though the structure function depends on Re, and the state of fully developed turbulence can be attained only asymptotically. This scenario has been termed "asymptotic Kolmogórov scaling." Given that K41 was predicated on the asymptotic condition $\operatorname{Re} \gg 1$, we believe that in its original formulation K41 was not meant to imply anything stronger than asymptotic Kolmogórov scaling. Hence, we characterize K41 and K62 as "asymptotic scenarios."

[14] Italics in the original.

[15] Reprinted in English in J. Nikuradse, NACA TM 1292 (1950).

[16] B. Knight and L. Sirovich, Phys. Rev. Lett. 65, 1356 (1990); T. S. Lundgren, Phys. Fluids 14, 638 (2002); and T. S. Lundgren, ibidem 15, 1074 (2003).

[17] L. D. Landau and E. M. Lifshitz, Fluid Mechanics 2nd edition, ch. III, p. 130 (Butterworth, Oxford, UK, 2000).

[18] G. I. Taylor, Proc. Roy. Soc. London A151, 421(1935).

[19] Reprinted in English in A. Strickler, Contribution to the question of a velocity formula and roughness data for streams, channels and close pipelines, translation by $\mathrm{T}$. Roesgen and W. R. Brownlie (Caltech, Pasadena, 1981).

[20] L. Prandtl, Essentials of Fluid Dynamics, 3rd edition, ch. III.11 (Blackie \& Son, London, 1953). The value $1 / 3$ of the exponent of $r / D$ in Strickler's expression can be derived by dimensional analysis from the value $2 / 3$ of the exponent of the hydraulic radius in Manning's empirical formula for the average velocity of the flow in a rough open channel. Manning obtained his formula independently of Strickler, on the basis of different experimental data. ; see G. Gioia and F. A. Bombardelli, Phys. Rev. Lett. 88, 014501 (2002).

[21] By "smooth conduit" we mean "mathematically smooth conduit." Whether an actual conduit may be considered mathematically smooth appears to be a question that grants no easy answers; see, e.g., G. I. Barenblatt and A. J. Chorin, Phys. Fluids 10, 1043 (1998); A. J. Smits and M. V. Zagarola, ibidem 10, 1045 (1998); A. E. Perry, S. Hafez, and M. S. Chong, J. Fluid Mech. 439, 395 (2001).

[22] A. Tsinober, An Informal Introduction to Turbulence, ch. 5 (Kluwer Academic Publishers, Dordrecht, The Netherlands, 2001).

[23] Most notably, the normalized dissipation in the experiments of D. P. Lathrop, J. Fineberg, and H. L. Swinney (Phys. Rev. A 46, 6390 (1992); Phys. Rev. Lett. 68, 1515 (1992)) and O. Cadot, Y. Couder, A. Daerr, S. Douady, and A. Tsinober (Phys. Rev. E 56, 427 (1997)). An extension of the analysis presented here might afford new insights into these experiments.

[24] For new work on our model of turbulent friction, see G. Gioia and P. Chakraborty, submitted to PRL, 2005. (Preprint is available at arXiv physics/0507066)

[25] Our schematic of Fig. 11 is not meant to represent the "d-type roughness" of A. E. Perry, W. H. Schofield, and P. N. Joubert, J. Fluid Mech. 37, 383 (1969). According to these authors, for this very specific type of roughness (square grooves normal to the mean flow) $f$ does not depend on $r$. Yet the distinction between d-type roughness 
and k-type roughness (the type of roughness in Nikuradse's pipes) appears to have been predicated on limited experimental data and must be regarded with caution; see J. Jiménez, Annu. Rev. Fluid Mech. 36, 173 (2004).
In any case, the schematic of Fig. 1 represents the rough walls of Nikuradse's experiments, and does lead to predictions that are in accord with those experiments. 